\title{
THE APRIL MEETING OF THE SAN FRANCISCO SECTION.
}

THE thirty-third regular meeting of the San Francisco Section was held at Stanford University on Saturday, April 5, with the chairman, Professor Cajori, presiding. The total attendance was twenty, including the following thirteen members of the Society:

Professor R. E. Allardice, Professor B. A. Bernstein, Professor Thomas Buck, Professor Florian Cajori, Professor M. W. Haskell, Professsor L. M. Hoskins, Professor D. N. Lehmer, Professor W. A. Manning, Professor H. C. Moreno, Dr. F. R. Morris, Professor C. A. Noble, Dr. Pauline Sperry, Dr. J. S. Taylor.

The next meeting of the Section will be held at the University of California, October 25, 1919.

The following papers were presented at the April meeting:

(1) Professor R. E. Allardice: "Note on the sextic with eight cusps."

(2) Professor Florian Cajori: "Observations on the development of algebraic notations."

(3) Dr. J. S. TAYloR: "A set of five postulates for Boolean algebras in terms of the operation 'exception."

(4) Dr. Pauline Sperry: "On the work of Gabriel Marcus Green in the field of projective differential geometry."

(5) Dr. Y. R. ChaO: "A note on 'continuous' mathematical induction."

(6) Professor L. M. Hoskins: "Effect of the asssumption of variable elasticity upon estimates of the rigidity of the earth."

(7) Professor W. A. Manning: "A fundamental theorem for simply transitive primitive groups."

(8) Professor E. T. BeLl: "On the number of representations of an integer as a sum of $3,5,7,9,11$, or 13 squares."

(9) Professor E. T. BELL: "On the number of representations of $2 n$ as a sum of $2 r$ squares."

(10) Dr. L. L. SmaIL: "Summability of double series."

Dr. Chao was introduced by Professor Bernstein. The papers of Professor Bell and Dr. Smail were read by title. Abstracts of the papers follow. 
1. In his note on the sextic with eight cusps, Professor Allardice finds the equation of this curve, and establishes the relations that connect the eight cuspidal points.

2. Professor Cajori observes that the adoption of algebraic notations is subject to two opposing forces: (1) The disinclination of mathematicians to memorize many symbols, (2) The convenience and power acquired from a well-chosen symbolism. In elementary algebra no large group of symbols invented by any one author has ever been bodily and permanently adopted; each symbol had its own struggle for existence, dependent not only upon merit but also upon particular configuration of circumstances. Bi-asymmetrical marks other than the numerals and letters of alphabets hardly ever survived. Notations have crossed national boundaries less easily than have principles and processes. There still exist side by side certain duplicate notations, rendering elementary algebra unnecessarily difficult. There is little precedent to indicate how much in the acquirement of desirable notations can be achieved by national and international organization.

3. Dr. Taylor presents a set of five comparatively simple postulates in terms of the operation "exception" with a proof of their consistency, independence, and sufficiency for the logic of classes. The complete existential theory of the five postulates is then developed, followed by a short discussion of the relative importance of the element 1 and negation (not-a). In order to show that each is as powerful as the other in one respect at least, a second set of postulates is presented in which, instead of postulating the element 1 and defining not- $a$, not- $a$ is postulated and the element 1 defined, a thing which curiously enough has never been done heretofore.

4. In a brief résumé, Dr. Sperry recited some of Dr. Green's more important contributions to the theory of projective differential geometry, emphasizing especially his work on $(a)$ the geometry of one-parameter families of space curves, and conjugate nets on a curved surface, and $(b)$ the general theory of curved surfaces and rectilinear congruences, as best illustrating the uniqueness of his analytic methods and the keenness of his geometric intuitions. 
5. Dr. Chao proves a theorem concerning a propositional function $\varphi(x)$ defined for a real interval and satisfying the following two conditions: (1) $\varphi(a)$ is true; (2) there exists a constant $\Delta$ in the interval such that $\varphi(x)$ implies $\varphi(x \pm \delta)$ for $0<\delta \leqq \Delta$.

6. Estimates of the rigidity of the earth are based upon the comparison of the actual yielding of the earth to tidal and centrifugal forces, as inferred from certain refined measurements, with the computed yielding of an elastic sphere having the dimensions and mass of the earth. The solution of this problem is known for a compressible sphere of uniform density, and for an incompressible sphere in which the density varies with distance from the center; the elastic moduli being in both solutions assumed to have uniform values throughout the body. Professor Hoskins has obtained the solution for a class of cases in which the elastic moduli as well as the density vary with distance from the center, and has obtained numerical results for a series of cases conforming closely with what is known regarding the actual properties of the earth. It is found that the known observational results are in close harmony with the computed strain of a sphere in which the elastic moduli are assumed to vary continuously from surface values based upon the known properties of rocks to values between six and seven times as great at the center. This would give a value of the modulus of rigidity at the center of about $1.70 \times 10^{12}$ C. G. S. units-about double the value for steel.

7. The theorem stated by Professor Manning is to this effect: If the subgroup that leaves one letter of a simply transitive primitive group fixed has a multiply transitive constituent, of degree $m$ say, then one of two things must occur; either all the transitive constituents of the subgroup that fixes one letter are multiply transitive groups of the same degree $m$ in simple isomorphism, or at least one transitive constituent of the maximal subgroup in question is of a degree greater than $m$ and a divisor of $m(m-1)$.

8. Defining as a primitive function of a positive integer $n$ one whose values may be calculated from the real divisors of $n$ alone, and as a simple function any sum of a finite number of primitive functions whose arguments form a recurring 
series of the second order, Professor Bell determines all cases in which the number of representations of an integer as a sum of $3,5,7,9,11$, or 13 squares is simple. The integers to be represented are classified first according to their linear forms modulo 8. For 3, 5, 7, 9 squares all possible cases are simple; for 11 or 13 squares only some are simple, and for 15, 17, 19, $21,23,25$ squares none are simple. The criteria deciding this seem to indicate non-simplicity for all odd numbers of squares $>25$; hence a special interest attaches to the cases treated in the paper. The resulting formulas are well adapted to numerical computation, and a comparison with the existing formulas for 5 or 7 squares (Eisenstein, H. J. S. Smith) shows a marked saving of labor in actual use. For 3,5 and 7 squares, and for some cases of 9,11 or 13 , the number of representations may be calculated by several rapid recurrences. An interesting by-product of the determination for 3 squares is a new derivation of the class number for a negative determinant by finite processes only. This leads to results, in finite form, which also easily yield numerical values, and which should be of use in the construction of tables beyond those existing, should the necessity for such arise.

9. In two papers Liouville (Journal de Mathématiques, series 2 , volume 6,1861 , pages 233,369 ) gave four general formulas relating to the number of representations of $2 n$ as a sum of $2 r$ squares. These contain as very special cases all the known instances in which such representations are either primitive or simple as defined in the preceding paper. Liouville suppressed both the proofs and the essential detail of showing how the undetermined coefficients, without knowledge of which his formulas cannot be used, may be found; these are supplied in Professor Bell's second paper. In proving the formulas it appears that similar general theorems exist for $2 n+1$ as a sum of $2 r$ squares, and that corresponding but less elegant results may be stated for both $2 n$ and $2 n+1$ as a sum of $2 r+1$ squares. Total and proper (as defined by Eisenstein) representations are treated by one analysis.

10. In a recent paper in the Annals of Mathematics (December, 1918), Dr. Smail has given a general method of summation for divergent series, based on a summation formula involving a function undefined except for certain restrictive 
conditions. In the present paper he extends this method to double series, and discusses the application of the method to convergent double series. It is found that the summation functions of the familiar methods of Cesàro, Hölder, Borel, LeRoy, Riesz, Vallee-Poussin, etc., can be used in building up summation formulas for double series.

B. A. Bernstein, Secretary of the Section.

\section{ON A CERTAIN GENERATION OF RATIONAL CIRCULAR AND ISOTROPIC CURVES.}

BY PROFESSOR ARNOLD EMCH.

(Read before the American Mathematical Society December 28, 1917.)

\section{Introduction.}

A CIRCUlar curve contains the circular points at infinity, or the isotropic points of the plane, as single, or as singular points. A plane isotropic curve is defined as a curve, all of whose infinite points are absorbed by the isotropic points. The equation of such a curve, which is necessarily of even order, in cartesian coordinates may be written in the form

$$
\left(x^{2}+y^{2}\right)^{k}+\varphi(x, y)=0,
$$

in which $\varphi(x, y)$ is a polynomial of degree $2 k-1$ at most.

If $P(\xi, \eta)$ is a fixed point and $A(x, y)$ any other point so that $P A=\rho$, and $\theta$ the angle between $P A$ and the positive direction of the $x$-axis, then the coordinates of $A$ are $x=\xi+\rho \cos \theta, y=\eta+\rho \sin \theta$, and satisfy equation (1) when $A$ is on the curve. The condition for this is an equation of the form

$$
\rho^{2 k}+\alpha_{1} \rho^{2 k-1}+\alpha_{2} \rho^{2 k-2}+\cdots+\alpha_{2 k-1} \rho+\alpha_{2 k}=0,
$$

in which $\alpha_{1}, \alpha_{2}, \cdots, \alpha_{2 k-1}$ are coefficients, which, in general, depend on $\xi, \eta, \theta$ and the coefficients of (1); while $\alpha_{2 k}$ is independent of $\theta$. The roots $\rho_{1}, \rho_{2}, \cdots, \rho_{2 k}$ of (2) are the distances $P A_{i}(i=1,2,3, \cdots, 2 k)$ of the points of inter- 